\title{
Concentration-alternating frequency response: A new method for studying polymer electrolyte membrane fuel cell dynamics
}

\author{
Antonio Sorrentino ${ }^{\mathrm{a}}$, Tanja Vidakovic-Koch ${ }^{\mathrm{a}}$, Richard \\ Hanke-Rauschenbach ${ }^{c}$, Kai Sundmacher ${ }^{a, b}$ \\ ${ }^{a}$ Max Planck Institute for Dynamics of Complex Technical Systems, Department Process \\ Systems Engineering, Sandtorstr.1, D-39106 Magdeburg, Germany \\ ${ }^{b}$ Otto-von-Guericke- Universität Magdeburg, Department Process Systems Engineering, \\ Universitätsplatz 2, D-39106 Magdeburg, Germany \\ ${ }^{c}$ Leibniz Universität Hannover, Electric Energy Storage Systems, Appelstr. 9A, D-30167, \\ Hannover, Germany
}

\begin{abstract}
In this work, the concentration-alternating frequency response analysis (cFRA) is proposed as a new method to study the dynamics of open electrochemical systems. The cFRA implies the perturbation of the electrochemical system through a feed characterized by a periodic concentration of a certain reactant. The most suitable detectable electrical output variable (potantial or current density) depends on the operating condition under which the experiment is performed. As illustrative example, a transient model accounting for the essential dynamic processes occurring in polymer electrolyte membrane fuel cells (PEMFC) is derived, and used to investigate the valuable insights obtainable from the cFRA spectra. Additionally, a sensitivity analysis is accomplished by use of sensitivity coefficients in order to estimate which model parameters can be better identified from EIS or from cFRA. It is shown that cFRA displayes a significantly
\end{abstract}


higher sensitivity than EIS for kinetic parameters. Moreover, a detailed analysis of the feasibility of the new technique is performed to identify appropriate operating conditions. In conclusion, cFRA can be regarded as an innovative and valuable tool for PEMFC identification.

Keywords: frequency response analysis, electrochemical impedance spectroscopy, PEM fuel cell dynamics, model based system identification, sensitivity analysis

\section{Introduction}

Frequency response analysis (FRA) is a well established method to investigate the dynamic behaviour of complex systems [1, 2]. It has been widely applied in many fields of science and enginering, as for istance, circuit analysis [3], engines assessment [4], aircraft flight dynamics [5], and diagnosis of electrochemical systems [6-29]. FRA implies the excitation of the system by a periodic input at different frequencies, and the study of a resulting output signal. The amplitude of the perturbation must be sufficiently small, so that the correlation input/output can be analyzed according to the simple mathemathical framework given by the linear system theory (LST). In this way, the algebraic or differential equations describing the non linear system are linearized, allowing to obtain even an analytical solution which is more easy to interpret. Additionally, the representation in frequency domain of the different dynamic measurements allow the discrimination and identification of the processes with different time constants.

The most used frequency response method in electrochemistry is the 
electrochemical impedance spectroscopy (EIS) [8, 15, 17-29]. It is based on the perturbation of the system through an electrical input, current or potential, and the detection of the complementary electric response. Electrochemical systems involve various transient phenomena characterized by different time constants, i.e. mass transport of the reagents and products in the different domains of the system, chemical and electrochemical intefacial reactions at the electrode interface, and adsorption of the reacting species. Electric stimulations are able to identify each one of these processes, and, furthermore they enable to explore a wide frequency bandwidth $\left(10^{6}-10^{-4} \mathrm{~Hz}\right)$. EIS has been applied to all kinds of electrochemical systems, as for instance, batteries, fuel cells, electrolysers, and corrosion systems.

However, time constants of the different transient phenomena could have comparable values, resulting in a strong coupling of different contributions $[7,8,15,17,20]$. Therefore, the attribution of the patterns observed in the impedance spectra is not always unambiguous, and a clear picture on the dynamics is not possible. For this reason, it has been suggested, in addition to EIS, to study system dynamics by applying nonelectrical inputs and/or outputs as well.

Tribollet et al. [8] and Collet-Lacoste [16] have reviewed and discussed the properties of most of the nonelectrical transfer functions proposed. Different transfer functions involving an electrical input and a nonelectrical output quantity have been suggested, as for example, electrogravimetric [7, 9] and electro-optical transfer functions [14]. Mostly, a nonelectrical input was considered, together with an electrical output. Of these, electro- 
hydro-dynamical impedance [6, 8], thermoelectrical impedance [10, 11], and photoelectrochemical impedance $[12,13]$ have been rather applied. Examples of transfer functions with both nonelectrical input and output are pressure modulated transfer functions, where modulated pressure has been used as an input, while the output was either the hydrogen flux coming out from a permeation membrane in the work of Bruzzoni [30], or the concentration of hydrogen at the interface of an elastic metal in the work of Zoltowski [31].

In this paper, a novel method, so called concentration-alternating frequency response analysis (cFRA), is proposed to investigate the dynamics of open electrochemical systems. In cFRA a periodic change of a reactant or even product concentration is applied as an input. As an output, the most suitable electric output variable (current or potential), depending on the condition under which the experiment is performed, has been considered. Somewhat similar idea has been proposed by Niroumand et al [32]. In their work, oscillations of potential and pressure in the cathode outlet flow, generated using feeds with low stoichiometry ratio, have been measured. A correlation between the frequency of the oscillations and time constants of water management phenomena has been assumed. However, the authors do not present a detailed proof about this conjecture.

As illustrative example, the complex case of the PEMFC is considered. PEMFCs dynamics are characterized by various processes simultaneously occurring in series and in parallel, i.e. electrochemical double layer charging at catalyst surface, gas and liquid transport in the gas diffusion electrode (GDE), water transport in the Nafion membrane [33-38]. 
For this system, the meaning of the EIS spectra patterns has been widely investigated in the past, through both theoretical and experimental works $[17,20-26,39,40]$. Despite these intensive efforts, due to the coupling of the different processes, the interpretation of the spectra is not clear in many experimental conditions [23, 26, 41, 42].

In this theoretical work, the potential of cFRA to study PEMFC dynamics has been evaluated. At first, a dynamic model of a PEMFC has been defined. To validate the model, experimental data from our lab have been used [27]. Moreover, a sensitivity analysis has been performed to evaluate the performances of the new transfer function with respect to parameter estimation. Finally, a detailed feasibility analysis has been carried out in order to identify appropriate operating windows for the cFRA.

\section{Theoretical background}

The theoretical background for the use of nonelectrical quantities to investigate the dynamics of electrochemical systems have been extensively investigated in the past. For example, a general mathematical framework to formulate and analyse different nonelectrical transfer functions has been proposed by Gabrielli and Tribollet [14]. A methodology to assess the reliability of the data collected through nonelectrical frequency response experiments has been developed by Macdonald [15]. Recently, ColletLacoste [16] has performed an analysis of the relationship between a generic

perturbation and a certain response in electrochemical systems based on the non-equilibrium thermodynamic theory. However, a procedure to relate the information obtanable from the frequency response spectra to the 
input and output considered has not been presented yet.

In this section, a mathematical framework to quantify the information contained in a certain transfer function regarding specific dynamics is presented. It is based on the definition of residual matrix. Furthemore, common formulations of cFRA for periodic concentration input, and potential or current outputs have been introduced.

\subsection{Canonical form of the state space representation and residual matrix}

Any set of mathematical equations describing the dynamic behaviour of a system, around a certain steady state point, can be reduced to the following linear matrix form

$$
\begin{aligned}
& \dot{X}=A X+B W \\
& Y=C X+D W
\end{aligned}
$$

where $\mathrm{A}, \mathrm{B}, \mathrm{C}$ and $\mathrm{D}$ respectively represent the so called state space, controllability, observability and transmission matrixes. It must be remarked that the values of the elements of the matrix A depends on the intrinsic dynamic features of the system, while, in the case of B and C, they are respectively functions of the kind input and output considered.

Generally, the system response to a perturbation is described through the corresponding transfer function in frequency domain, obtained by applying the Fourier transform to the system of equations (1)-(2). It reads

$$
H(i \omega)=\frac{\tilde{Y}(i \omega)}{\tilde{W}(i \omega)}=C\left(i \omega I_{n}-A\right)^{-1} B+D
$$


For $\Lambda=\operatorname{diag}\left(\lambda_{i}\right) \quad(i=1,2 \ldots n)$ be the diagonal matrix of the eigenvalues $\lambda_{i}$ of $\mathrm{A}, \mathrm{P}$ the matrix of the corresponding left eigenvectors $p_{i}$, and $Q^{T}=P^{-1}$ the matrix of the corresponding right eigenvector $q_{i}^{T}$, the equivalent canonical form of the system (1)-(2) reads [2, 4]:

$$
\begin{aligned}
\dot{X}_{d} & =\Lambda X_{d}+Q^{T} B W \\
Y & =C P X_{d}+D W .
\end{aligned}
$$

According to this new representation, the transfer function (3) can also be represented as

$$
H(i \omega)=C P\left(i \omega I_{n}-\Lambda\right)^{-1} Q^{T} B+D=\sum_{k=1}^{n} \frac{r_{k}}{i \omega-\lambda_{k}}+d_{1}
$$

where

$$
r_{k}=C p_{k} q_{k}^{T} B
$$

The quantities $r_{k}$ represent the elements of the so defined residual matrix, each one relative to a certain dynamic state variable $i$ characterized by a time constant $\tau_{i}=-\frac{1}{\lambda_{i}}$. Basically, the magnitude of the terms of the residual matrix provides a measure of the importance of the contribution of the corresponding variable on the dynamics in a certain input/output system description. Therefore, the residual matrix is a valuable theoretical tool to estimate which kind of dynamics influence most the frequency response spectra. It can be used for model reduction purpose as well, suggesting which variable can be neglected in describing the effect of a certain perturbation. 
In the next sections, the concept of residual matrix is used to compare the performances of different transfer functions formulated to study various dynamics in electrochemical systems. The main focus is about verifying the selectivity of the selected transfer function to some specific transient process.

2.2. Concentration-alternating frequency response

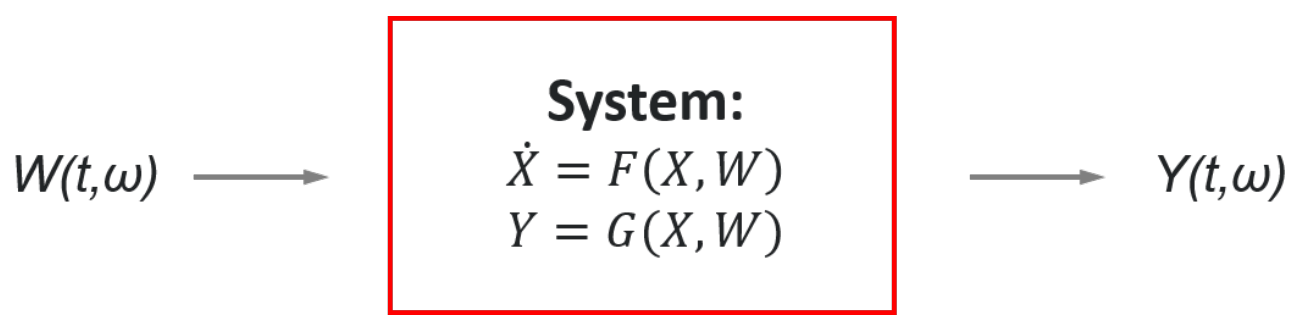

Figure 1: General scheme of cFRA experiment.

For an open electrochemical system (see figure 1), fed by a periodic concentration input $(W(t, \omega)))$, two frequency response functions can be formulated by considering potential or current as outputs. These concentrationalternating frequency response transfer functions (cFRTFs) are defined as follows

$$
\begin{aligned}
\xi_{V}(i \omega) & =\frac{\Delta I(i \omega)}{\Delta C_{\alpha}(i \omega)} \\
\xi_{I}(i \omega) & =\frac{\Delta V(i \omega)}{\Delta C_{\alpha}(i \omega)} .
\end{aligned}
$$

\section{PEM Fuel Cell Model}

A schematic representation of the structure and of the different compartments which constitute a PEM fuel cell is displayed in figure 2. Ac- 


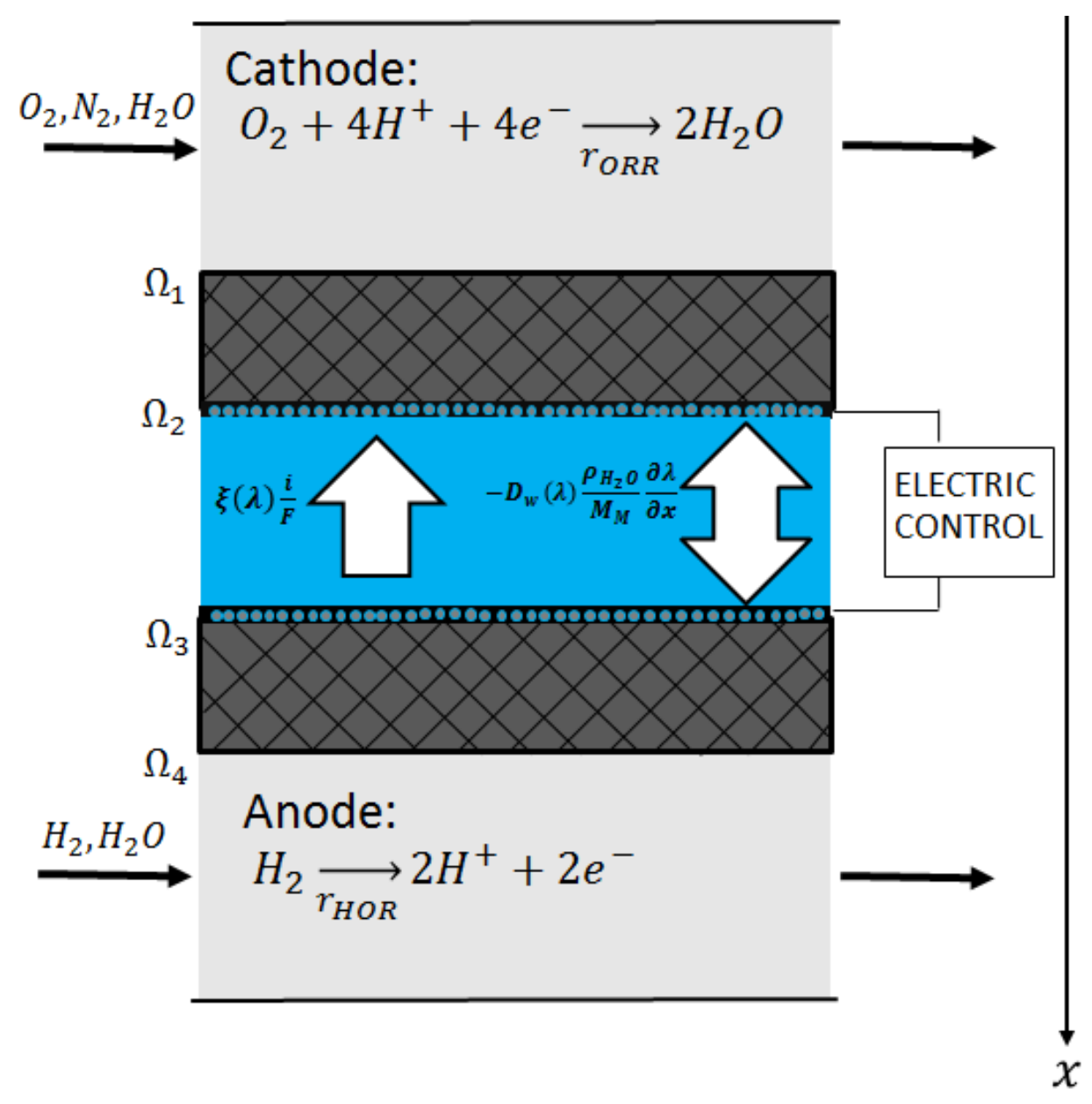

Figure 2: Scheme of a PEM fuel cell.

cording to the literature [33-38], the physical processes which influence the transient performance of a PEM fuel cell are the following: (i) the charging (or discharging) of the double layer at the electrode surface, (ii) the mass transport of the gaseous reactants and of the produced liquid water on the cathode side of the cell, (iii) the water transport and the resulting hydration of the Nafion membrane. 
In the following model description, all main dynamic phenomena described above have been considered except for the transport of the liquid water in the channel $(\mathrm{CH})$ and gas diffusion layer (GDL) of the cathode. This assumption reduces the validity of the model to those operating conditions where the water condensation effects are negligible, as for example at moderate operating current densities and at not fully humidified flows. According to Eikerling et al [43] below $0.7 \mathrm{~A} / \mathrm{cm}^{2}$, at an operating temperature of $298 \mathrm{~K}$, and low humidification conditions all water produced in the catalyst layer evaporates before entering into the GDL. Based on this study, one can assume that simulations of PEMFC experiments at operating currents below this value will assure the reliability of the hypotesis of negligible liquid water in the system. It should be also mentioned that the temperature contemplated in the present study is $343 \mathrm{~K}$, which shifts the amount of current at which the liquid water appears in the GDL to even higher current densities. Additional evidence for low level of liquid water formation under present conditions (high feed stoichiometry with a relative humidity of $80 \%$, atmospheric pressure, $26 \mathrm{~cm}^{2}$ active area, and current density range up to $0.4 \mathrm{~A} / \mathrm{cm}^{2}$ ), can be extracted from diverse literature studies where the formation of the liquid water was investigated by neutron imaging technique [44,45]. For example, based on[45] one can estimate that only 3\% of the total volume of GDL (void fraction) and channels is occupied by liquid water under present conditions.

Dynamic mass balances have been formulated for all the chemical species contained in all the compartments of the cell. Concentration variations along the sandwich coordinate $x$ are accounted, while along the axial coor- 
dinate $y$ the composition is considered constant. Additionally, isothermal and isobaric conditions are assumed inside the cell. The isobaric assumption is based on the estimated pressure drop inside the cathode channel of $0.01 \%$ (please see the Supplementary Information).

To relate the effect of the different transport phenomena to the electric responses, a dynamic charge balance at the catalyst surface has been developed. Realistic boundary conditions connect the dynamics of the different compartments of the cell have been formulated.

In the following subsections, more detailed information about the model equations for the different domains of the cell is presented.

\subsection{Mass balances in channel and gas diffusion layer}

The channels are treated as a perfectly mixed compartments of volume $\mathrm{V}$, with inner gas composition equaling the outlet gas composition. In these compartments, the sink term is represented by the gas flux $J_{\alpha}$ entering the GDL at the interfaces $\Omega_{1}$ and $\Omega_{4}$ (see figure 2). Consequently, the dynamic mass balance for a generic chemical compound $\alpha$ in the $\mathrm{CH}$ in terms of partial pressure $P_{\alpha}$ reads

$$
V \frac{\mathrm{d} P_{\alpha}^{C H}}{\mathrm{~d} t}=P_{\alpha, i n}^{C H} F_{i n}-P_{\alpha}^{C H} F_{o u t}+\left.R T A J_{\alpha}\right|_{\Omega_{i}, t} \quad i=1,4 .
$$

where the symbol $A$ stands for the area of the reactive surface of the electrode, while $F_{\text {in (out })}$ are the volumetric flows of reactants or products of the fuel cell reactions. The meaning of the other symbols can be found in the List of Symbols. 


\begin{tabular}{|c|c|c|}
\hline Quantity & Value & Source \\
\hline Gas flow rate at cathode $F_{i n}^{c}\left(\mathrm{~m}^{3} \mathrm{~s}^{-1}\right)$ & $9.24 \times 10^{-6}$ & [27] \\
\hline Gas flow rate at anode $F_{i n}^{a}\left(\mathrm{~m}^{3} \mathrm{~s}^{-1}\right)$ & $5.85 \times 10^{-6}$ & [27] \\
\hline Total pressure $P_{\text {tot }}(\mathrm{Pa})$ & $10^{5}$ & \\
\hline Oxygen pressure of the cathode inlet $P_{\mathrm{O}_{2}, \mathrm{in}}^{\mathrm{CH}, \mathrm{c}}(\mathrm{Pa})$ & $1.58 \times 10^{4}$ & [27] \\
\hline Water pressure of the cathode inlet $P_{\mathrm{H}_{2} \mathrm{O}, \mathrm{in}}^{\mathrm{CH}}(\mathrm{Pa})$ & $2.48 \times 10^{4}$ & [27] \\
\hline Hydrogen pressure of the anode inlet $P_{\mathrm{H}_{2}, i n}^{\mathrm{CH}, a}(\mathrm{~Pa})$ & $7.51 \times 10^{4}$ & [27] \\
\hline Geometric surface area of the catalyst $A\left(\mathrm{~m}^{2}\right)$ & $2.6 \times 10^{-3}$ & [27] \\
\hline Double layer capacitance for cathode and anode $C_{d l}\left(\mathrm{~F} \mathrm{~m}^{-2}\right)$ & 100 & \\
\hline Temperature $T(\mathrm{~K})$ & 343 & [27] \\
\hline Dew point temperature of the inlet gases $T(\mathrm{~K})$ & 338 & [27] \\
\hline Reference concentration $\mathrm{O}_{2} C_{\mathrm{O} 2}^{r e f}\left(\mathrm{~mol} \mathrm{~m}^{-3}\right)$ & 40 & [46] \\
\hline Product of specific catalyst area and exchange current $a i_{0}\left(\mathrm{~A} \mathrm{~m}^{-3}\right)$ & $10^{4}$ & [46] \\
\hline Cathode catalyst layer thickness $t_{c c l}(\mathrm{~m})$ & $10^{-5}$ & [46] \\
\hline Kinetic constant for hydrogen oxidation $K_{H O R}\left(\mathrm{~mol} \mathrm{~m}^{-2} \mathrm{~s}^{-1}\right)$ & $4.15 \times 10^{-1}$ & [46] \\
\hline Kinetic constant for hydrogen adsorption $K_{a d, H_{2}}(\mathrm{~Pa})$ & $5.07 \times 10^{4}$ & [46] \\
\hline Charge transfer coefficient at the anode $\alpha_{a}$ & 0.5 & \\
\hline Charge transfer coefficient at the cathode $\alpha_{c}$ & 0.38 & \\
\hline Porosity of the GDL $\epsilon$ & 0.7 & [34] \\
\hline Effective diffusivity for $\mathrm{O}_{2} D_{\mathrm{O}_{2}, \mathrm{~N}_{2}}^{\text {eff }}\left(\mathrm{m}^{2} \mathrm{~s}^{-1}\right)$ & $9.85 \times 10^{-6}$ & \\
\hline Effective diffusivity for $\mathrm{H}_{2} \mathrm{O} D_{\mathrm{H}_{2} \mathrm{O}, \mathrm{N}_{2}}^{e f f}\left(\mathrm{~m}^{2} \mathrm{~s}^{-1}\right)$ & $6.7 \times 10^{-6}$ & \\
\hline Volume of the channel $V\left(\mathrm{~m}^{3}\right)$ & $1.74 \times 10^{-6}$ & [27] \\
\hline Open circuit potential $U_{o c p}(\mathrm{~V})$ & 1.2 & \\
\hline
\end{tabular}

Table 1: List of input parameters 
The main mean of transport in the GDL is diffusion. While most models in the past considered only diffusive transport in the GDL [47], newer works include also the convective flow [48]. Based on latter works, the contribution of the convective flow depends on the geometry of flow fields. This issue has been also discussed by Benziger et al [49], who concluded that the oxygen flux should include both convective and diffusive contribution. They derived the equation having the same form as Ficks law, showing that the convection will be dominant only at very low nitrogen concentrations. Assuming air cathode stream one can estimate that the main mean of transport in GDL will be diffusion. The diffusion flux of the ternary $\mathrm{O}_{2}-\mathrm{N}_{2}-\mathrm{H}_{2} \mathrm{O}$ mixture is usually described by the Maxwell-Stefan approach. However, the Ficks law describes pretty well this ternary system as well, when humified air is used as oxidant [50]. Therefore, the mass balance for the GDLs reads

$$
\frac{\partial P_{\alpha}^{G D L}}{\partial t}=D_{\alpha, \beta}^{e f f} \frac{\partial^{2} P_{\alpha}^{G D L}}{\partial x^{2}}
$$

where $\beta$ stands for the nitrogen on the cathode side. The effective diffusivity $D_{\alpha, \beta}^{e f f}$ is expressed in accordance to the Bruggeman correlation

$$
D_{\alpha, \beta}^{e f f}=\epsilon^{1.5} D_{\alpha, \beta} .
$$

Because of the assumption of negible liquid water in the porous electrode, the porosity $\epsilon$ is considered constant along the layer. For this reason, the diffusivity constant figures outside the differential term on the right side of the equation (11). 
The catalyst layer is considered as an interface. Therefore, the rates of the electrochemical reactions appear only in the corresponding boundary conditions illustrated in the next sections.

\subsection{Nafion membrane}

A single phase formulation based on the Springer approach [39] is used to describe the dynamics of the Nafion membrane. Therefore, the water

content in the membrane $\lambda_{M}$, which is defined as the ratio between the number of water molecules and the number of sulfonic sites in the membrane, is considered as dependent variable. The relationship between the water concentration in the Nafion $\left(C_{W}\right)$ and $\lambda_{M}$ is

$$
C_{W}=\frac{\rho_{M}}{M_{M}} \lambda_{M}
$$

where $\rho_{M}$ and $M_{M}$ are the density and the molecular weight of the Nafion membrane respectively. Then, according to the equation (3), the dynamic mass balance for the water in the membrane can be expressed as follows

$$
\frac{\rho_{M}}{M_{M}} \frac{\partial \lambda_{M}}{\partial t}=-\frac{\partial N_{W}}{\partial x}
$$

As stated in different studies $[47,51]$, the water flow $N_{W}$ is generated through three different mechanisms: (i) diffusion due to the water activity gradient along the cell, (ii) hydraulic permeation driven by the gradient of capillary pressure, and (iii) the electrosmotic flow due to the water 
molecules dragged by the protons migrating from the anode to the cathode side. Altough both diffusive and hydraulic permeation types of water transport contribute concurrently to the water transport, the one of the two mechanisms will dominate depending on $\lambda_{M}$ values. It is generally accepted that the diffusive way of transport will dominate at low $\lambda_{M}$ values while the hydraulic at high $\lambda_{M}$ values. Yet, there is a disagreement $[47,51]$, about the critical $\lambda_{M}^{*}$ value which marks the transition from diffusive to hydraulic type of water transport (for example, the critical $\lambda_{M}^{*}$ value of 3 is suggested by Eikerling et al [51], while much higher value of 14 is suggested by Weber and Newman [47]). According to Eikerling et al [51], by following the similarity with the diffusive term, the hydraulic permeation term can be expressed in terms of water content gradient by introducing an effective diffusivity coefficient. Furthermore, by assuming tha validity of electroneutrality hypotesis in the membrane, so that the proton flow can be considered equal to the current density, one can write:

$$
N_{\lambda}=-\left(D_{\text {diff }}^{e f f}\left(\lambda_{M}\right)+D_{\text {hyd }}^{e f f}\left(\lambda_{M}\right)\right) \frac{\partial \lambda_{M}}{\partial x}-\xi\left(\lambda_{M}\right) \frac{i}{F}
$$

where $D_{\text {diff }}^{e f f}$, and $D_{\text {hyd }}^{e f f}$ are effective diffusivities for two different mechanisms of water transport. Having this in mind, and in order to implicitly account for both types of transport mechanisms, by keeping at the same time the level of model complexity low, the equation (15), for the water flux in combination with a semi-empirical expression [52] for effective water diffusivity in the Nafion membrane was used. The disavantage of this simplification is that the effect of the pressure gradient on the water transport in the membrane cannot be described by the model. Given these as- 
sumptions, $N_{W}$ reads

$$
N_{W}=-D_{W}\left(\lambda_{M}\right) \frac{\rho_{M}}{M_{M}} \frac{\partial \lambda_{M}}{\partial x}-\xi\left(\lambda_{M}\right) \frac{i}{F} .
$$

The dependences of the water diffusivity in the membrane $\left(D_{W}\left(\lambda_{M}\right)\right)$ and of the electrosmotic drag constant on the water content $\left(\xi\left(\lambda_{M}\right)\right)$ are respectively expressed by the following relations taken from Fuller [52] and Springer et al. [39]

$$
\begin{aligned}
D_{W}\left(\lambda_{M}\right) & =\lambda_{M} D_{W}^{0} \exp \left(-\frac{2436}{T}\right) \\
\xi\left(\lambda_{M}\right) & =K_{M} \lambda_{M} .
\end{aligned}
$$

The equation (17), which express the diffusivity, is a semi-empirical relation derived by fitting of the experiments displayed in the work of Zawodzinski et al [53].

The potential loss due to the ohmic resistance related to the Nafion membrane is strongly dependent on the total water content. To account for this effect, the potential loss $\Delta \Phi_{M}$ is calculated by integrating the local electric field along the membrane

$$
\Delta \Phi_{M}=i \int_{0}^{l_{M}} \frac{1}{\kappa_{M}\left(\lambda_{M}\right)} \mathrm{d} x .
$$

The membrane conductivity $\kappa_{M}$ is expressed as function of the water content by the following relation [39]

$$
\kappa_{M}=\left(0,005139 \lambda_{M}-0,00326\right) \exp \left(1268\left(\frac{1}{303}-\frac{1}{T}\right)\right)
$$




\subsection{Charge balance}

Charge balances at the catalyst layers interfaces have been formulated by considering the double layers acting as capacitors in parallel to charge transfer resistances due to the electrochemical kinetic limitation. The resulting equations for both anode and cathode sides read

$$
\begin{aligned}
& i=C_{d l}^{c} \frac{\mathrm{d} \eta_{c}}{\mathrm{~d} t}-4 F r_{O R R} \\
& i=C_{d l}^{a} \frac{\mathrm{d} \eta_{a}}{\mathrm{~d} t}+2 F r_{H O R} .
\end{aligned}
$$

The variable $\eta$ stands for the overpotential, while $C_{d l}$ is the capacitance of the double layer. The balance is formulated according to the reaction stoichiometry shown in figure 2. Because of the assumption of the fast transport in the $\mathrm{CL}$, the charge balance is not spatially distributed. The kinetic expressions for the oxygen reduction $r_{O R R}$ and hydrogen oxidation $r_{H O R}$ reactions are approximations of the Butler-Volmer equation [46, 54, 55]

$$
\begin{aligned}
& r_{\mathrm{ORR}}=\frac{P_{\mathrm{O}_{2}}^{C L}}{R T C_{\mathrm{O}_{2}, \text { ref }}} t_{c c l} \frac{a i_{0}}{4 F} \exp \left(-\frac{\alpha_{c} F}{R T} \eta_{c}\right) \\
& r_{\mathrm{HOR}}=\frac{K_{\mathrm{HOR}}\left(P_{\mathrm{H}_{2}}^{C L}\right)^{1 / 2}}{2 \sqrt{K_{\mathrm{H}_{2}, a d}}} \sinh \left(\frac{\alpha_{a} F}{R T} \eta_{a}\right) .
\end{aligned}
$$

The meaning of the terms in kinetic expressions can be read in the List of the Symbols.

To relate the potential of the cell to the different losses due to the various mass transport limitations and electrokinetic resistances, a polarization equation is formulated 


$$
U=U_{O C P}-\eta_{a}+\eta_{c}-\Delta \Phi_{M}
$$

where $U_{O C P}$ is the open circuit potential. The current density $i$ is implicitly given in the equation (25), so that this relation can be used to determine the total potential or the current erogated by the cell depending on the electric control we consider, i.e. galvanostatic (fixed current) or voltastatic (fixed potential).

\subsection{Boundary conditions}

The developed model consists of six second order partial differential equations (PDE): five spatially distributed mass balances for all the components of the gas mixture in the GDLs (three for the cathode and two for the anode side), and an additional PDE related to the water transport in the membrane. Therefore, the formulation of twelve boundary conditions $(B C)$ is required to solve the set of equation.

As shown in the figure 2, the interfaces between the gas CHs and GDLs are labelled $\Omega_{1}$ ans $\Omega_{4}$, respectively standing for the cathode and the anode part. The continuity of the partial pressure beween these two domains is chosen as boundary condition, so that we obtain

$$
\left.P_{\alpha}^{G D L}\right|_{x=\Omega_{1}, t}=P_{\alpha}^{C H},\left.\quad P_{\alpha}^{G D L}\right|_{x=\Omega_{4}, t}=P_{\alpha}^{C H} .
$$

As mentioned in the previous sections, the catalyst layer is considered as an interface where the reaction occurs as soon as the reactants reach it. 
On the cathode, the oxygen diffusion equals the rate of the oxygen consumption. Therefore, the $\mathrm{BC}$ for the oxygen pressure reads

$$
-\left.\frac{D_{\mathrm{O}_{2}, a i r}^{e f f}}{R T} \frac{\partial P_{\mathrm{O}_{2}}^{G D L}}{\partial x}\right|_{x=\Omega_{2}, t}=r_{\mathrm{ORR}}
$$

Since nitrogen does not participate to the ORR, the gas is stagnant, and the following condition is valid

$$
-\left.\frac{D_{N_{2}, a i r}^{e f f}}{R T} \frac{\partial P_{N_{2}}^{G D L}}{\partial x}\right|_{x=\Omega_{2, t}}=0
$$

For the water case, the steady balance at the catalyst layer is formulated taking into account the water produced by the ORR, the one incoming by the electrosmotic flow, and the water coming out through the diffusion through the Nafion membrane. Then, the following equation is obtained

$$
\left.\frac{-D_{\mathrm{H}_{2} \mathrm{O}, a i r}^{e f f}}{R T} \frac{\partial P_{\mathrm{H}_{2} \mathrm{O}}^{G D L}}{\partial x}\right|_{x=\Omega_{2}, t}-\left.D_{W}\left(\lambda_{M}\right) \frac{\rho_{M}}{M_{M}} \frac{\partial \lambda_{M}}{\partial x}\right|_{x=\Omega_{2, t}}=2 r_{O R R}+\xi\left(\lambda_{M}\right) \frac{i}{F} .
$$

On the anode, analog assumptions have been contemplated. According to that, the $\mathrm{BC}$ for the partial pressure of hydrogen reads

$$
-\left.\frac{D_{\mathrm{H}_{2}, \mathrm{H}_{2} \mathrm{O}}^{e f f}}{R T} \frac{\partial P_{\mathrm{H}_{2}}^{G D L}}{\partial x}\right|_{x=\Omega_{3}, t}=r_{H O R}
$$


Since, there is no water produced by the HOR, the water crossing the membrane from the cathode side by diffusion and electrosmotic flow is the only flux to be accounted in the balance. Then, the following balance is formulated

$$
-\left.\frac{D_{\mathrm{H}_{2} \mathrm{O}, \mathrm{H}_{2}}^{e f f}}{R T} \frac{\partial P_{\mathrm{H}_{2} \mathrm{O}}^{G D L}}{\partial x}\right|_{x=\Omega_{3}, t}=-\left.D_{W}\left(\lambda_{M}\right) \frac{\rho_{M}}{M_{M}} \frac{\partial \lambda_{M}}{\partial x}\right|_{x=\Omega_{3}, t}+\xi\left(\lambda_{M}\right) \frac{i}{F} .
$$

At the catalyst/Nafion interface, the water vapour is considered in equilibrium with the water inside the Nafion membrane. According to that, as BCs for the water balance in the Nafion membrane, the isothermal water uptake equation is considered on both sides [39]:

$$
\lambda_{W}=0,043+17,\left.81 \frac{P_{\mathrm{H}_{2} \mathrm{O}}^{C L}}{P_{\text {sat }}}\right|_{x=\Omega_{i}, t}-39,\left.85 \frac{P_{\mathrm{H}_{2} \mathrm{O}}^{C L}}{P_{\text {sat }}}\right|_{x=\Omega_{i}, t} ^{2}+\left.36 \frac{P_{\mathrm{H}_{2} \mathrm{O}}^{C L}}{P_{\text {sat }}}\right|_{x=\Omega_{i}, t} ^{3}
$$

where $i=3,4$.

\section{Results and discussion}

The model compiled in the previous section has been descretized, and numerically solved in MATLAB ${ }^{\circledR}$ enviroment at different steady state conditions. Then, it has been further linearized around steady state points in order to obtain a linear state space system representation (eq. 1-2). After that, the desired transfer functions have been calculated with a help of the equation (3) avoiding nonlinear contributions and without concerning 
about the amplitude of the perturbation applied (please see Supplementary Information for further clarification).

First, classic EIS spectra have been simulated and validated by experimental data from our lab (all details of the experiments have been provided in the experimental work of Kadyk et al [27]). Then, cFRA spectra obtained by oxygen or water partial pressure perturbations at the cathode inlet have been plotted at the same operating conditions for comparison purpose. The different insights obtainable from the spectra of the two different experimental techniques are discussed through different examples. Additionally, a sensitivity analysis with respect to some specific parameters has been performed in order to compare the performances of the EIS and cFRA in the task of parameter estimation. In the last part, the experimental feasibility and the limits of the new frequency response technique have been examined.

\subsection{Model validation}

In order to verify the capability of the model to predict the dynamics of a PEM fuel cell, EIS spectra have been simulated and compared to the experimental ones shown in the work of Kadyk [27]. The experimental conditions and the values of the parameters used in the model are listed in the table 1. In the figures $3 \mathrm{a}-\mathrm{c}$, the experimental spectra measured at three different working points, i.e. $0.115 \mathrm{~A} \mathrm{~cm}^{-2}, 0.231 \mathrm{~A} \mathrm{~cm}^{-2}$ and 0.346 $\mathrm{A} \mathrm{cm}^{-2}$, have been shown. These conditions correspond roughly to three different regimes of a PEMFC operation: activation controlled region, linear region controlled by the ohmic resistance due to the membrane, and a region in which the mass transport resistances start to have a more sig- 

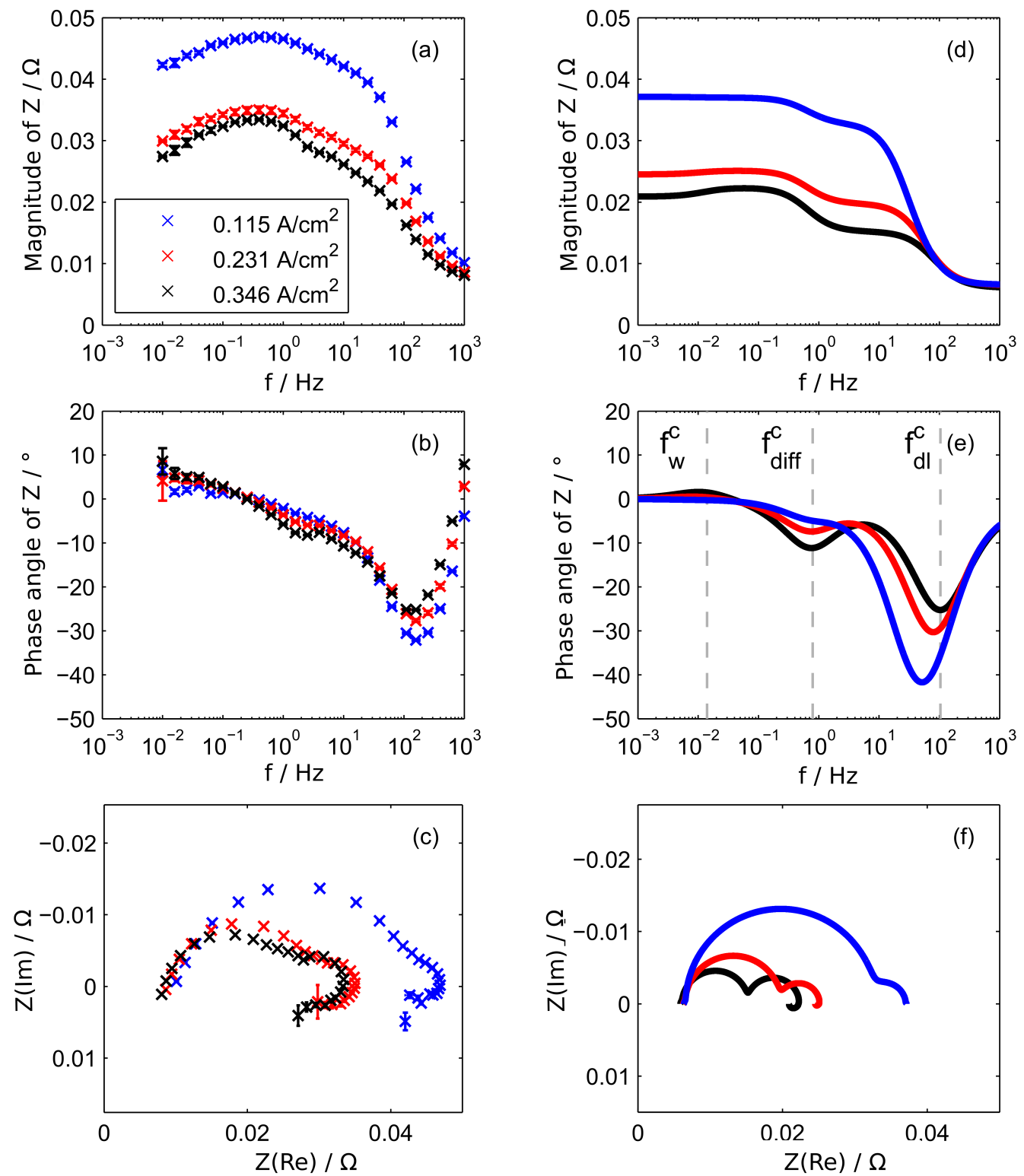

Figure 3: EIS spectra collected at 3 different working points. (a)-(c) experimental spectra, (d)-(e) simulated spectra.

nificant influence. As shown in the figures $3 \mathrm{~d}-\mathrm{f}$, the model reproduces well the experimental results, predicting the effect of the dominant tran- 
sient processes on the spectra. Basically, three phenomena dominate the dynamics of the cell, which characteristic frequencies, and, consequently, the time constants can be determined by the turning points in the phase angle diagram or by the inflection points in the magnitude Bode plot representation [18]. As illustrative example, characteristic frequencies related to the phase angle plot at the highest studied steady state current density are shown in the figure $3 \mathrm{e}$. The effect of the kinetic charge transfer resistance acting in parallel with the double layer dynamics is observed in the high frequency region $\left(f_{d l}^{c}=105 \mathrm{~Hz}\right)$. It is usually the fastest transient observed in PEM fuel cells $[17,20]$. At a frequency around $0.7 \mathrm{~Hz}$, termed as $f_{\text {diff }}^{c}$, the dynamics related to the gas transport inside the $\mathrm{CH}$ and GDL on the cathode side are detected. As shown also in other works, the contribution of the mass transport on the anode side to the spectra is too small to be distinguished $[17,20]$. Finally, in the low frequency range (larger response times) the characteristic frequency for the water transport in the membrane $\left(f_{w}^{c}=0.01 \mathrm{~Hz}\right)$ can be observed [17, 20, 23, 26, 42]. It should be mentioned that the present spectra are relatively easy to interpret, since the observed dynamics do not overlap. As it was mentioned in the previous sections, in many cases, the overlapping of different phenomena occurs in the spectra, making difficoult the investigation of the system dynamics.

\section{2. cFRA spectra}

For comparison purposes, cFRA spectra obtained by oxygen partial pressure perturbations, under voltastatic control, and at the same operating conditions previously considered for the EIS have been simulated. The results are displayed in figure 4 . In the magnitude plot (figure 4a), 
two inflections poinst are observed at the same characteristic frequencies previously related to the gas diffusion in the $\mathrm{CH}$ and GDL domains, and to the water transport in the membrane.

The dynamics associated to the double layer charging is not visible at high frequencies. This can be rationalized by the time delay between the input signal perturbation (oxygen concentration change) and the system response in terms of current and overpotential changes. This time delay reflects also in the phase angle plot (figure $4 \mathrm{~b}$ ) at high frequencies where the phase angle is increasing monotonously over the angle of $360^{\circ}$, reaching the plateau of $1080^{\circ}$ at very high frequencies (see the inset of the figure 4b). Additionally, the Nyquist plots exhibit a spyral at high frequencies (see the inset of the figure 4c). It can be further assumed that this delay should be equal to the time required for the gas to diffuse inside the cathode $\mathrm{CH}$ and GDL, and reach the catalyst surface $\left(\tau=\frac{1}{f_{d i f f}^{c}}=0.22 s\right)$. This assumption can be further demonstrated as follows.

The time delay $\Delta t$ of the output is related to the frequency $\omega_{c}$ at which in the Nyquist plot the imaginary part turns for the first time negative (see the inset of figure 4c) according to the following equation [56]

$$
\Delta t=\frac{\pi-\tan ^{-1} \omega_{c}}{\omega_{c}} .
$$

The calculated time delay by the equation (33) based on data in figure $4 \mathrm{c}$ is $0.24 \mathrm{~s}$, which agrees well with the value estimated based on the characteristic time constant for the diffusion in the $\mathrm{CH}$ and GDL of $0.22 \mathrm{~s}$. It follows further that the time delay will be influenced by mass transfer conditions in the $\mathrm{CH}$ and the GDL, where the characteristic frequency $\omega_{c}$ 

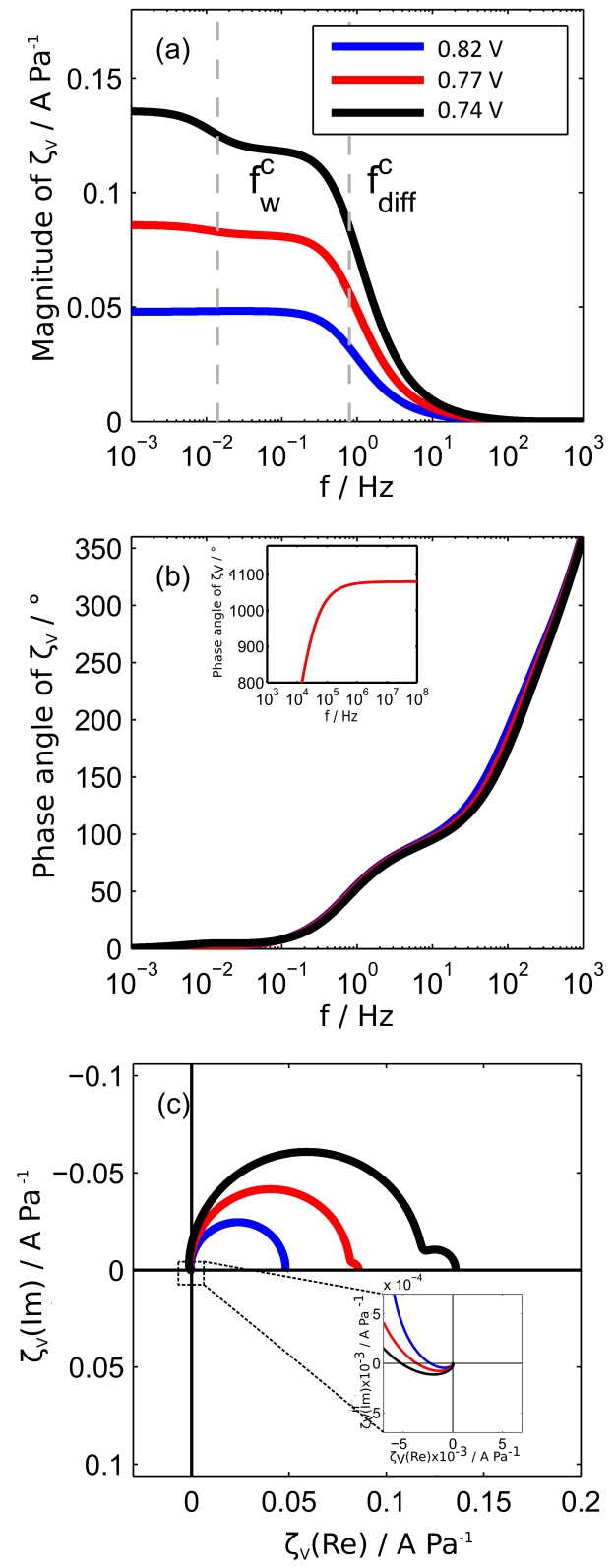

Figure 4: Simulated cFRA spectra obtained by oxygen partial pressure perturbations under voltastatic control at 3 different working points. 
will be dependent on the gas diffusivity, GDL porosity or its thickness.

In figure $5 \mathrm{a}$, the influence of oxygen diffusivity on cFRA spectra has been shown in a Nyquist plot representation. As one can see, the cFRA spectra are sensitive to the change of this parameter. The figure $5 \mathrm{~b}$ shows zoomed high frequency area of the cFRA spectra. As can be seen, the value of the highest frequency intercept on the real axis increases as the diffusivity decrease $\left(\omega=60.1 \mathrm{~Hz}, \omega^{\prime}=51.48 \mathrm{~Hz}, \omega^{\prime \prime}=43.96 \mathrm{~Hz}\right)$.

This, in accordance to the equation (33), results in the associated time delays of $\Delta t=0.26 \mathrm{~s}, \Delta t^{\prime}=0.31 \mathrm{~s}$, and $\Delta t^{\prime \prime}=0.36 \mathrm{~s}$. An increase of the time delay with a decrease of diffusivity is expected, since a lower diffusivity means a slower gas diffusion.

The magnitude of cFRA obtained under voltastatic control (solid line), and under galvanostatic control (dashed line) are compared in the figure 6. Unlike the voltastatic case, the galvanostatic spectra do not display any change in the response at the characteristic frequency for the transport of water in the membrane. The only present turning point is the one revealing the gas transport dynamics. This is because the galvanostatic control implies a costant reaction rate and, consequently, a costant water production by the ORR on the cathode. It results in a steady hydration of the membrane which is not a dynamic element under this operating condition, and give a constant contribution to the ohmic resistance of the cell.

This supposition is further reflected in figure 7 , where simulations of oxygen pressure step experiments (figure 7a) are displayed. Specifically, the electric responses to the pressure stimulation under voltastatic and galvanostatic conditions are reported (figure $7 \mathrm{~b}$ ) together with the dynamic 

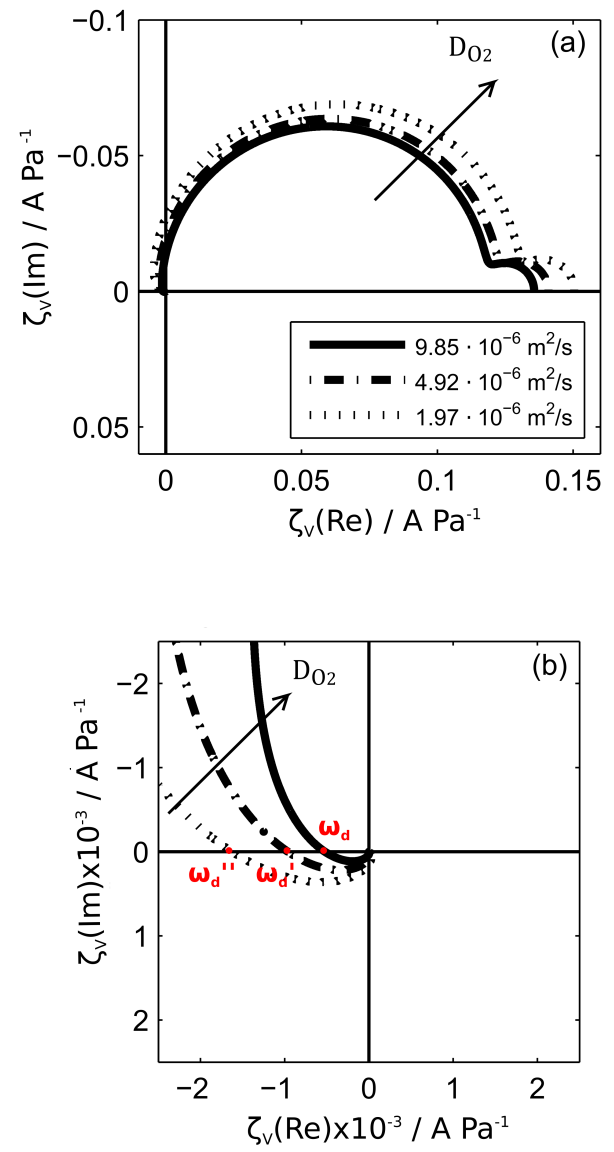

Figure 5: Simulated cFRA Nyquist plot obtained by oxygen partial pressure perturbations under voltastatic control at 3 different values of oxygen diffusivity. Operating current: $0.346 \mathrm{~A} \mathrm{~cm}^{-2}$.

behaviour of the ohmic losses due to the membrane (figure 7c), calculated by the equation (19). Under voltastatic control (figure $7 \mathrm{~b}$, solid line), the current increases from its initial steady state value to the intermediate new value in a less than $1 \mathrm{~s}$. At a bit longer time scale $(t>1 \mathrm{~s})$, additional increase of current can be observed, with a new steady state value reached in ca. 30s. The first change of the current at $t<1 \mathrm{~s}$ can be attributed to the 


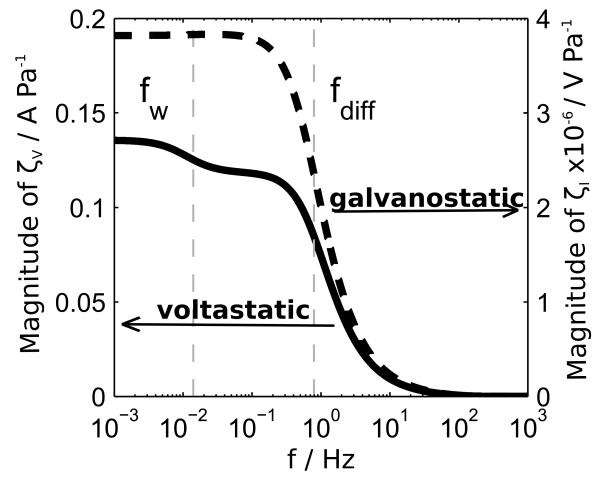

Figure 6: Comparison of cFRTF in magnitude Bode plots obtained by oxygen partial pressure perturbations under voltastatic (solid line) and galvanostatic (dashed line) control. Operating current of $0.346 \mathrm{~A} \mathrm{~cm}^{-2}$.
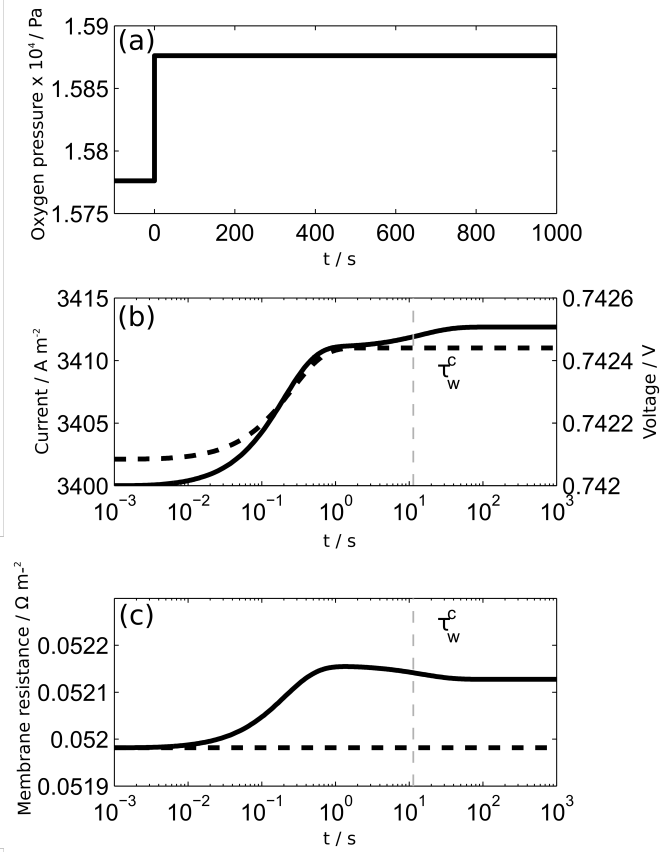

Figure 7: Pressure step simulations under voltastatic (solid line) and galvanostatic (dashed line) conditions. 
change in the oxygen concentration due to the pressure step. The increase at $t>1 s$ is not directly related to the concentration change. This increase can be explained by a change of a membrane resistance caused by additional water transport in the membrane. The decrease of the membrane resistance under voltastatic conditions leads to an increase of the activation overpotential (cathode overpotential will be mainly influenced), causing an increase of the current density. This is further demonstrated in the figure $7 \mathrm{c}$ (solid line), where the change of the membrane resistance over time has been shown. At first, the membrane resistance is increasing (due to the current increase), but at $t>10 \mathrm{~s}$ it starts to decrease. This time corresponds to the time constant of water diffusion in the membrane. On the contrary, the potential response stays constant at the same range of time under galvanostatic control, as well as the ohmic resistance which value does not change during the time course of the experiment (see figure $7 \mathrm{~b}-\mathrm{c}$, dashed line). This confirms the previous speculation about the constant hydration of membrane by keeping the current constant. The present discussion suggests that the comparison between the cFRA spectra collected under galvanostatic and voltastatic conditions can be used to identify and discriminate the contribution of the membrane dynamics to the frequency response spectra.

In the figure 8 , the simulated magnitude of cFRA spectra attained by water partial pressure perturbations are reported. In these simulations, the steady state water pressure was significantly below the saturation point. For both electric responses, the spectra selectively detect the dynamics associated to the water transport in the membrane. The only characteristic 


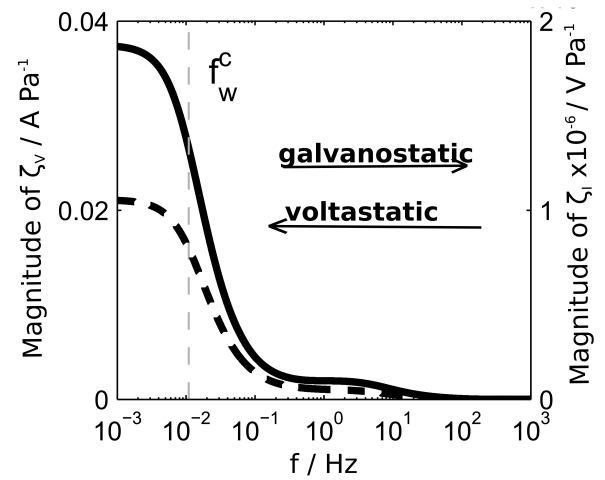

Figure 8: Comparison of the magnitude of cFRA in Bode plot representation obtained by water partial pressure perturbations under voltastatic (solid line) and galvanostatic (dashed line) control. Operating current of $0.346 \mathrm{~A} \mathrm{~cm}^{-2}$.

frequency determinable is indeed the one corresponding to this phenomena. A slight turning point is observed in the frequency range of the transport in the GDL. It suggests that water perturbation can be used to study selectively the dynamics related to the Nafion membrane or, more generally, to the the phenomena connected to the water transport in the cell.

\begin{tabular}{lcccc}
\hline Technique & $r_{D L}^{r e l}(\%)$ & $r_{G D L, \mathrm{O}_{2}}^{r e l}(\%)$ & $r_{\mathrm{CH}, \mathrm{O}_{2}}^{r e l}(\%)$ & $r_{W}^{r e l}(\%)$ \\
\hline EIS & 49,24 & 0,18 & 38,24 & 8,27 \\
$\mathrm{c}\left(\mathrm{O}_{2}\right)$ EFRA (pot) & 2,8 & 0,98 & 88,09 & 6 \\
$\mathrm{c}\left(\mathrm{O}_{2}\right)$ EFRA (gal) & 2,05 & 0,64 & 96,61 & 0,49 \\
$\mathrm{c}\left(\mathrm{H}_{2} \mathrm{O}\right)$ EFRA (pot) & 1,61 & 2,05 & 0,05 & 87,5 \\
$\mathrm{c}\left(\mathrm{H}_{2} \mathrm{O}\right)$ EFRA (gal) & 1,06 & 0,65 & 0,02 & 91,15
\end{tabular}

Table 2: Relative residual values. Operating current: $0.346 \mathrm{~A} \mathrm{~cm}^{-2}$.

The previous analysis demonstrates that cFRA capability to discriminate between different dynamic processes is dependent not only on the 
chosen concentration input perturbation, but also on the electric control conditions of the experiment (galvanostatic or voltastatic). This conclusion follows also from analysis of the terms of the residual matrix (eq. 7) for all the studied frequency response methods. To rationalize the information contained in the spectra, the relative contribution of the residual $r_{i}$ has been calculated through the following equation

$$
r_{i}^{r e l}=100 \frac{r_{i}}{\sum_{i=1}^{n} r_{i}} .
$$

In the table 2 , the values of the relative residuals $r_{i}^{\text {rel }}$ for model variables related to the accounted dynamic processes have been determined, where, for example, $r_{D L}^{r e l}$ stands for the relative residual related to the double layer charging, $r_{G D L, O}$ rel for the oxygen transport in the GDL, $r_{\mathrm{CH}, \mathrm{O}_{2}}^{r e l}$ for the oxygen transport in the $\mathrm{CH}$, and $r_{M}^{r e l}$ for the transport of the water in the membrane. The relative terms are expressed in percentage. As claimed in the previous sections, the higher the value of the relative residual, the higher the impact of the dynamic process on the spectra. The dynamics of variables characterized by a value of the residual below 3\% are not observed in the spectra. In the case of EIS, the values of the residuals are significant for most involved dynamic processes. An exception is the gas diffusion in the GDL, which value of the relative residual is below $1 \%$. These calculations agrees with the EIS spectra previously displayed, in which insights for all the main dynamics have been observed. It is noticeable that the diffusion inside the GDL has not given a great contribution to all the spectra analyzed. The relative residuals related to the cFRA spectra obtained by oxygen perturbation are characterized by a negligible value 
for the double layer transient under both the operating modi. Indeed, this process could not be identified in the cFRA spectra. Specifically, for the cFRA under galvanostatic condition, the only significant relative residual is the one which stand for the dynamics of the diffusion in the channel, influencing the $96 \%$ of the spectra. This is reflected in the magnitude spectra (figure 6) discussed above as well. Finally, the selectivity of the cFRA spectra obtained by water pressure perturbation regarding the transport in the Nafion membrane is also verified. The values of the relative residual $r_{W}^{r e l}$ corresponding to this phenomena are around the $90 \%$, while the others are all below the $3 \%$. Therefore, the residual matrix can be considered as valuable theoretical instrument to predict the contributions of the different dynamics to a spectra of a certain transfer function.

\subsection{Sensitivity analysis}

To evaluate the sensitivity of the different frequency response techniques towards a certain parameter, the normalized local sensitivity coefficients are defined as follows

$$
\left|S_{\theta}(\omega)\right|=\frac{\theta}{H(\omega)} \frac{\partial H(\omega)}{\partial \theta} .
$$

In general, a high sensitivity implies a smaller variance in the measure of a certain parameter, resulting in a more correct estimation $[57,58]$. Therefore, in the present context, the value of the sensitivity coefficient represents a comparative term with respect to the quality of the parameter estimation performed using different experimental techniques. 


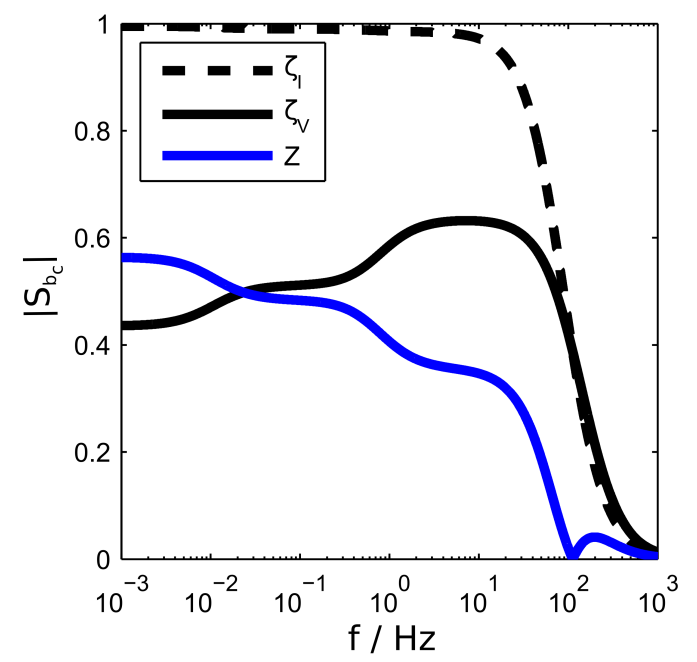

Figure 9: Comparison between sensitivity coefficients related to the cathodic transfer coefficient $\alpha_{c}$. Operating current of $0.346 \mathrm{~A} \mathrm{~cm}^{-2}$.

In the figure 9, the sensitivity coefficients with respect to the cathodic transfer coefficient $\alpha_{c}$, which is related to the Tafel slope of the ORR $\left(b_{c}\right)$, are shown. The Tafel slope is a key parameter to investigate the performance of a catalyst and, therefore, plays an important role with respect to the efficiency of a fuel cell. The voltastatic cFRTF obtained by oxygen stimulations has a sensitivity comparable to the one of the classic EIS. On the other hand, excellent results have been accomplished for the galvanostatic spectra, which sensitivity coefficient is ca. two times higher than the sensitivity coefficients of other two methods in almost whole frequency range. It suggests that this experimental method is more reliable than the classic EIS for the estimation of the Tafel slope, and, consequently, for parameters correlated to the kinetics of the ORR.

A further advantage of the use of the galvanostatic operating mode can be deduced from the figure 10. Sensitivity coefficients for the Tafel slope, 


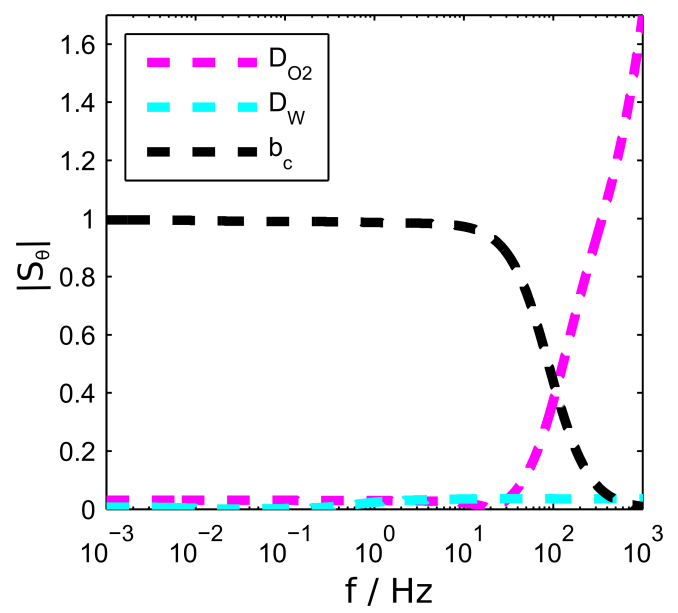

Figure 10: Comparison of sensitivity coefficients related to Tafel slope (dashed black line), oxygen diffusivity (pink dashed line), and diffusivity of water in the membrane (cyan dashed line), for galvanostatic cFRA magnitude spectra obtained by oxygen perturbations. Operating current: $0.346 \mathrm{~A} \mathrm{~cm}^{-2}$.

oxygen diffusivity, and water diffusivity in the membrane are reported together, in order to understand the influences of the the resistances (kinetic, gas transport, and water transport in Nafion) to the cFRA spectra. For the frequency range from $10^{-3}$ to $10 \mathrm{~Hz}$, the oxygen and water diffusivity sensitivities are negligible compared to the Tafel slope sensitivities. This result implies two conclusions: (i) the magnitude of the galvanostatic cFRA spectra is mainly affected by the ORR kinetics, (ii) the kinetic parameters are not correlated to the other parameters. Specifically, the latter assertion attests that the uncertainties of other parameters do not affect the kinetic parameters, allowing a more correct estimation about them. This additional finding suggests further the suitability of the use oxygen perturbation under galvanostatic control to study the ORR kinetics in a PEM 
fuel cell.

\subsection{Feasibility analysis}

As for all linear frequency response techniques, in order to collect reliable data, cFRA experiments must respect conditions of linearity $[2,14$, 15]. This mainly implies the application of pressure perturbation with an amplitude sufficiently small to avoid nonlinearities in the electric response of the system. On the other hand, the amplitude of the pressure should also be large enough to produce an electric perturbation which could be detected and distinguished from the eventual noise. Therefore, the amplitude of a proper input is restricted by the requirement of linearity on one side, and the signal detectability on the other side. Therefore, in the following, detecatability and nonlinearity limitations specific for the considered electric output are defined.

Several methods to quantify the nonlinearities in the output of a frequency response have been proposed [1,59]. As a most convenient method, in the present case a so-called total harmonic distortion (THD) of a signal has been introduced. This method has been successfully applied in the past $[60,61]$. Given a periodic signal $E$ composed by different harmonics of amplitude $\delta_{i}$, the THD is defined as

$$
T H D=100 \sqrt{\frac{\delta_{1}^{2}+\delta_{2}^{2}+\ldots}{\delta_{1}}} .
$$

As suggested in different works [60,61], for sinusoidal inputs, the THD value should not exceed 5 . Over this value, the contents on non linearities 

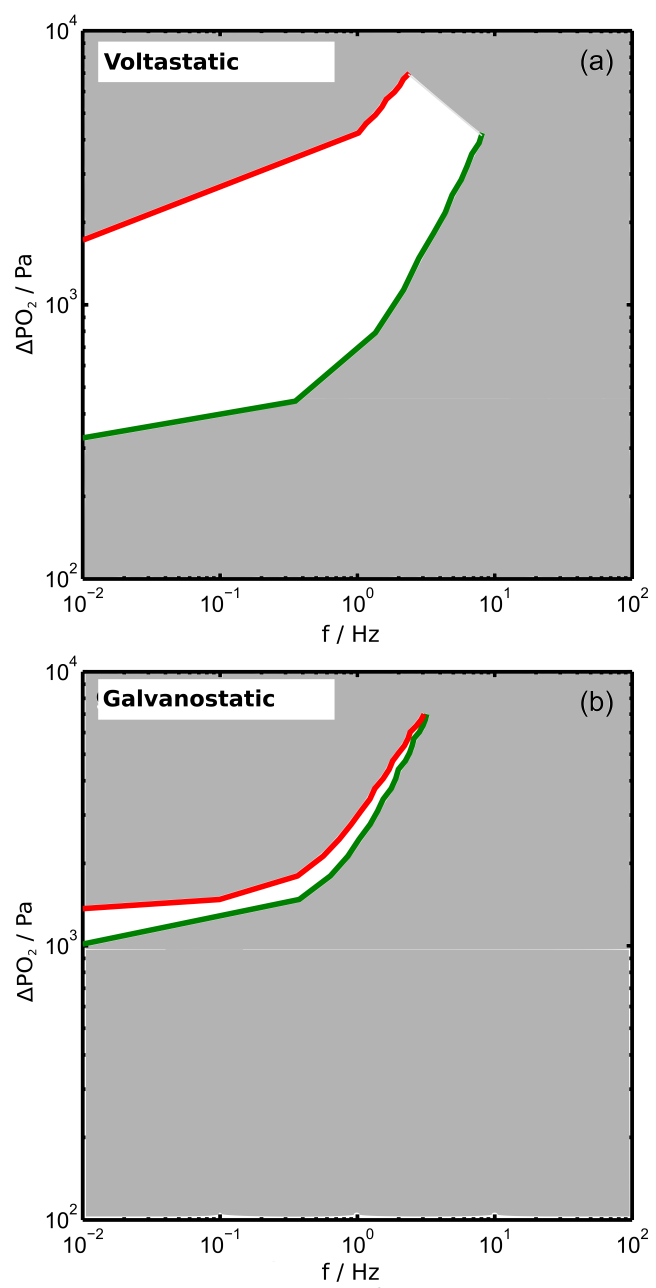

Figure 11: Operating window for cFRA experiments involving oxygen perturbation under voltastatic (a), and galvanostatic (b) control. Operating current: $0.346 \mathrm{~A} \mathrm{~cm}^{-2}$.

is supposed to be too high to perform a data analysis conform with the LST.

The detectability limit is restricted by the detectability of the electric response. The minimum value of the amplitude which ca be clearly detected is fixed at $5 \mathrm{~mA}$ and $5 \mathrm{mV}$, respectively for the voltastatic, and galvanostatic operating modi. These detectability limits are largely overestimated, 
since the modern potentiostats have a resolution $0.3 \mu \mathrm{V}$ for the potential response. However, a more conservative approach is reasonable for a theoretical study.

To theoretically verify the respectability of the cFRA technique to the imposed limitations, the model has been numerically solved to simulate the electric response of the cell to sinusoidal oxygen pressure perturbations at different frequencies, and with amplitudes of different magnitudes. For each output, the amplitude and the THD have been collected. By treating these data, the operating windows in figure 11, for voltastatic and galvanostatic control, have been designed. Each point of the diagram is related to an input specification, i.e. an amplitude of oxygen pressure and a frequecy which characterize a perturbation. The green line indicates the detectability limit. The inputs characterized by the specification points below this line are not able to generate a detectable electric response. Likewise, the input with the specifications indicated by the points over the nonlinearity limit (red line) produce an output containing a not tolerable quantity of nonlinearities. Therefore, the white region represent the operating window for the cFRA which satisfies the feasibility requirements. It is noticeable that the voltastatic cFRA present a wider feasible operating window than the galvanostatic one. However, in both the cases, oxygen pressure perturbations able to produce linear and detectable responses are possible.

\subsection{Possible contribution to the spectra of other dynamics}

As stated in the previous sections, the catalyst layer has been considered as an interface neglecting all the dynamics related to this layer. 
Some of these missing transients could considerably affect cFRA spectra. Above all, pressure variations could influence the water management in the porous structure of the catalyst layer. In fact, at different pressures, water saturation limit, capillary pressures, and evaporation rate change influencing the water distribution inside the porous structure of the layer[43, 62]. Water creates contacts between catalytic Pt particles and Nafion influencing the electrochemical active surface area available for the oxygen reduction. Moreover, it must be remarked that the current model could not describe correctly the water transport in the membrane in conditions under which hydraulic permeation is the dominating mechanism. In this circumstances, changes of pressure would affect the value of the capillary pressure at the membrane interface altering the water distribution [51]. This phenomena could add additional dynamics which are not accounted in the current status of the model.

In order to study PEMFC dynamics under different operating conditions than in the present work (for example high humidity feeds, high current densities ecc.), these phenomena should be included in the dynamic model since they can have significant influence on cFRA spectra.

\section{Conclusions}

A new frequency response method to analyze the transient behaviour of open electrochemical systems has been introduced. It is based on the stimulation of the system through a feed characterized by a periodic concentration of a certain reactant, and on the measure of the resulting electric response, current or potential, which depends on the control modi (poten- 
tiostatic or galvanostatic). As an illustrative example, the potential of the novel techniques has been theoretically investigated by a PEMFC model.

It has been shown that cFRA spectra can discriminate the different dynamic processes occurring in the cell, depending on the kind of concentration perturbation, and electric control applied. The comparison between the cFRA spectra obtained by oxygen perturbations under voltastatic and galvanostatic controls, can be used to identify the contribution of the transport of water in the membrane to the system dynamics. Moreover, this phenomena can be selectively studied through water perturbation sent to the cell.

The results of the sensitivity analysis attest that the use of pressure perturbations under galvanostatic mode allow a more correct estimation of the kinetic parameters, compared to the classic EIS.

Additionally, the feasibility analysis demostrates that cFRA experiments for PEM fuel cells could be performed respecting the linearity constraints.

The results of the cFRA simulations are going to be validated through experiments currently performed in our lab. A more advanced version of the model accounting additional phenomena is in progress.

\section{Acknowledgments}

The authors gratefully acknowledge the financial support from the German Federal Ministry of Education and Research (BMBF) under the project GECKO, Grant No. 03SF0454B. 


\section{Notation}

List of symbols

$a_{W} \quad$ Water activity,-

$A \quad$ Area of the electrode, $\mathrm{m}^{2}$

$\mathrm{Cdl} \quad$ Double layer capacity, $\mathrm{F} \mathrm{m}^{-2}$

$D_{i, a i r} \quad$ Diffusivity in the air, $\mathrm{m}^{2} \mathrm{~s}^{-1}$

$D_{w} \quad$ Diffusivity of water in Nafion, $\mathrm{m}^{2} \mathrm{~s}^{-1}$

$F \quad$ Faraday constant, $\mathrm{C} \mathrm{mol}^{-1}$

$F_{\alpha} \quad$ Volumetric flow, $\mathrm{m}^{3} \mathrm{~s}^{-1}$

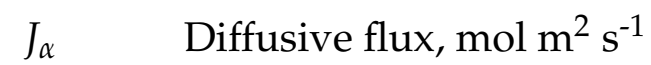

$k_{M} \quad$ Electrosmotic flow constant

$K_{H 2, a d}$ Hydrogen adsorption constant, $\mathrm{Pa}$

$K_{H, o x} \quad$ Hydrogen oxidation constant, mol m ${ }^{-2} \mathrm{~s}^{-1}$

$i \quad$ Current density, $\mathrm{A} \mathrm{m}^{-2}$

$i_{0} \quad$ Exchange current density, $\mathrm{A} \mathrm{m}^{-2}$

$M_{M} \quad$ Molecular weight of the membrane, $\mathrm{Kg} \mathrm{m}^{-3}$

$N_{w} \quad$ Water flux through the membrane, $\mathrm{mol} \mathrm{m}^{-2} \mathrm{~s}^{-1}$

$P_{\alpha} \quad$ Partial pressure, $\mathrm{Pa}$

$r_{H O R} \quad$ Reaction rate of hydrogen oxidation reaction, $\mathrm{mol} \mathrm{m}^{-2} \mathrm{~s}^{-1}$

$r_{O R R}$ Reaction rate of oxygen reduction reaction, $\mathrm{mol} \mathrm{m}^{-2} \mathrm{~s}^{-1}$

$R \quad$ Universal gas constant, mol J-1 $\mathrm{K}^{-1}$

$t_{c c l} \quad$ Catalyst layer thikness, $\mathrm{m}$

$V \quad$ Volume of the channel, $\mathrm{m}^{3}$

U Potential, V

$x \quad$ Sandwich coordinate, $\mathrm{m}$ 


$\begin{array}{ll}y & \text { Axial coordinate, } \mathrm{m} \\ \alpha & \text { Charge transfer coefficient, - } \\ \eta & \text { Overpotential, } \mathrm{V} \\ \kappa_{M} & \text { Conductivity of the membrane, } \mathrm{S} \mathrm{m}^{-1} \\ \lambda & \text { Water content, - } \\ \xi & \text { Electrosmotic constant, - } \\ \rho_{\mathrm{H}_{2} \mathrm{O}} & \text { Water density, } \mathrm{kg} \mathrm{m}^{-3} \\ \text { Superscripts } & \\ a & \text { Anode } \\ c & \text { Cathode } \\ C H & \text { Channel } \\ C L & \text { Catalyst layer } \\ e f f & \text { Effective value } \\ G D L & \text { Gas diffusion layer } \\ M & \text { Nafion membrane }\end{array}$

Subscripts

ccl Cathode catalyst layer

$d l \quad$ Double layer

HOR Hydrogen oxidation reaction

in Incoming

$O R R$ Oxygen oxidation reaction

out Outcoming

w Water

$\alpha \quad$ General component 


\section{References}

[1] R. Pintelon and J. Schoukens. System identification: a frequency domain approach. Wiley-IEEE Press, 1980.

[2] Z. Gajic. Linear dynamic systems and signals. Prentice Hall, 1980.

[3] K. S. Suresh Kumar. Electric circuits and networks. Pearson Education, 2008.

[4] M. Athans, P. Kapasouris, E. Kappos, and H. A. Spang. Linearquadratic gaussian with loop-transfer recovering methodology for the f-100 engine. Journal of Guidance Control and Dynamics, 9(1):45 $52,1986$.

[5] C. L. Seacord. Modeling transport in polymer-electrolyte fuel cells. Journal of the Aeronautical Sciences, 17(8):481 - 498, 1950.

[6] K. Tokuda, S. Bruckenstein, and B. Miller. The frequency response of limiting current to sinusoidal speed modulation at a rotating disk electrode. Journal of Electrochemical Society, 122(10):1316 - 1321, 1975.

[7] C. Gabrielli. Identification of electrochemical processes by frequency response analysis. Schlumberger Farnborough, 1980.

[8] B. Tribollet. The modulated flow at a rotating disk electrode. Journal of Electrochemical Society, 130(10):2016 - 2026, 1983. 
[9] S. Bourkane, C. Gabrielli, and M. Keddam. Study of electrochemical phase formation and dissolution by ac quartz electrogravimetry. Electrochimica Acta, 34(8):1081 - 1092, 1989.

[10] J. L. Valdes and B. Miller. Thermal modulation of rotating disk electrode: frequency and transient response. Journal of Electrochemical Society, 135(9):2223 - 1231, 1988.

[11] A. Olivier, E. Merienne, J. P. Chopart, and O. Aaboubi. Thermoelectrochemical impedances 1 . A new experimental device to measure thermoelectrochemical transfer functions. Electrochimica Acta, 37(11):1945 - 1950, 1992.

[12] H. Song and D. D. Macdonald. Photoelectrochemical impedance spectroscopy: validation of the transfer function by Kramers-Kronig transformation. Journal of Electrochemical Society, 138(5):1408 - 1410, 1991.

[13] A. Goossens and D. D. Macdonald. A photoelectrochemical passive study of passive tungsten. Journal of Electroanalytical Chemistry, 352(1):65 - 81, 1993.

[14] C. Gabrielli and B. Tribollet. A transfer function approach for a generalized electrochemical impedance spectroscopy. Journal of the Electrochemical Society, 141(5):1147 - 1146, 1994.

[15] D. D. Macdonald, E. Sikora, and G. Engelhardt. Characterizing electrochemical systems in the frequency domain. Electrochimica Acta, 43(1):87 - 107, 1998. 
[16] J. R. Collet-Lacoste. The electrochemical impedance spectroscopy and associated transfer functions: non-equilibrium thermodynamics consideration. Electrochimica Acta, 49(5):4967 - 4977, 2004.

[17] X. Yuan, H. Wang, J. C. Sun, and J. Zhang. Ac impedance technique in PEM fuel cell diagnosis - a review. Internationa Journal of Hydrogen Energy, 32(7):4365 - 4380, 2007.

[18] M. Orazem and B. Tribollet. Electrochemical impedance spectroscopy. Wiley, 2008.

[19] B. Y. Chang and S. M. Park. Electrochemical impedance spectroscopy. Annual Reviews of Analytical Chemistry, 3(5):207 - 229, 2010.

[20] S. M. Rezaei Niya and M. Hoorfar. Study of proton exchange membrane fuel cells using electrochemical impedance spectroscopy technique - a review. Journal of Power Sources, 240(8):281 - 293, 2013.

[21] T. D. Springer, T. A. Zawodzinski, M. S. Wilson, and S. Gottesfeld. Characterization of polymer electrolyte fuel cells using ac impedance spectroscopy. Journal of the Electrochemical Society, 143(2):587 - 599, 1996.

[22] A. Aricò, V. Antonucci, V. Alderucci, E. Modica, and N. Giordano. Ac impedance spectroscopy study of oxygen reduction at Nafion coated gas-dissusion electrodes in sulfuric acid: Teflon loading and methanol cross-over effects. Journal of Applied Electrochemistry, 23:1107 $-1116,1993$. 
[23] T. J. P. Freire and E. R. Gonzalez. Effect of membrane characteristics and humidification conditions on the impedance response of polymer electrolyte fuel cells. Journal of Electroanalytical Chemistry, 503(1):57 68, 2001.

[24] O. Antoine, Y. Bultel, and R. Durand. Oxygen reduction reaction kinetics and mechanism on platinum nanoparticles inside Nafion. Journal of Electroanalytical Chemistry, 499(1):85 - 94, 2001.

[25] A. A. Franco and M. Tembely. Transient multiscale modeling of aging mechanisms in PEMFC cathode. Journal of the Electrochemical Society, 154(7):B712 - B723, 2007.

[26] K. Wiezell and P. Gode G. Lindberg. Steady state and EIS investigations of hydrogen electrodes and membrane in polymer electrolyte fuel cells. Journal of the Electrochemical Society, 153(4):A749 - A758, 2006.

[27] T. Kadyk, R. Hanke-Rauschenbach, and K. Sundmacher. Nonlinear frequency response analysis of PEM fuel cells for diagnosis of dehydration, flooding and co-poisoning. Journal of Electroanalytica Chemistry, 630:19-27, 2009.

[28] T. Kadyk, R. Hanke-Rauschenbach, and K. Sundmacher. Nonlinear frequency response analysis for the diagnosis of carbon monoxide poisoning in PEM fuel cell anodes. Journal of Applied Electrochemistry, 41(9):1021 - 1032, 2011. 
[29] T. Kadyk, R. Hanke-Rauschenbach, and K. Sundmacher. Nonlinear frequency response analysis of dehydration phenomena in polymer electrolyte membrane fuel cells. Journal of Hydrogen Energy, 37(9):7689 $-7701,2012$.

[30] P. Bruzzoni, R. M. Carranza, and J. R. Collet-lacoste. A pressure modulation to study surface effects in hydrogen permeation through iron base. Electrochimica Acta, 44(24):4443 - 4452, 1999.

[31] P. Zoltowski. Concentration transfer function of hydrogen diffusion in self-stressed metals. Journal of the Electroanalytical Chemistry, 512(1):64-73, 2001.

[32] A. M. Niroumand, W. Merida, M. Eikerling, and M. Safi. Pressurevoltage oscillations as diagnostic tool for PEFC cathode. Electrochemistry Communications, 12(1):122 - 124, 2010.

[33] C. Y. Wang. Fundamental models for fuel cells engineering. Chemical Reviews, 104(10):4727 - 4766, 2004.

[34] Y. Wang and C. Y. Wang. Transient analysis of polymer electrolyte fuel cells. Electrochimica Acta, 50(6):1307 - 1315, 2005.

[35] Y. Wang and C.-Y. Wang. Dynamics of polymer electrolyte fuel cells undergoing load changes. Electrochimica Acta, 51(19):3924 - 3933, 2006.

[36] A. Z. Weber and J. Newman. Modeling transport in polymerelectrolyte fuel cells. Chemical Reviews, 104(10):4679 - 4726, 2004. 
[37] J. Benziger, E. Chia, J.F. Moxley, and I.G. Kevrekidis. The dynamic response of PEM fuel cells to changes in load. Chemical Engineering Science, 60(6):1743 - 1759, 2005.

[38] P. R. Pathapati, X. Xue, and J. Tang. A new dynamic model for predicting transient phenomena in a PEM fuel cell system. Renewable Energy, 30(1):1 - 22, 2005.

[39] T. E. Springer, T. A. Zawodzinski, and S. Gottesfeld. Polymer electrolyte fuel cell model. Journal of the Electrochemical Society, 138(8):2334 $-2342,1991$.

[40] A. A. Franco, P. Schott, C. Jallut, and B. Maschke. A dynamic mechanistic model of an electrochemical interface. Journal of the Electrochemical Society, 153(6):A1053 - A1061, 2006.

[41] V. A. Paganin, C. L. F. Oliveira, E. A. Ticianelli, T. E. Springer, and E. R. Gonzalez. Modelistic interpretation of the impedance response of a polymer electrolyte fuel cell. Electrochimica Acta, 43(24):3761 3766, 1998.

[42] B. Andreaus, A. J. McEvoy, and G. G. Scherer. Analysis of performance losses in polymer electrolyte fuel cells at high curren density by impedance spectroscopy. Electrochimica Acta, 47(13-14):2223 $-2229,2002$.

[43] M. Eikerling. Water management in cathode catalyst layers of PEM fuel cells: a structure-based model. Electrochimica Acta, 53(3):E58 E70, 2006. 
[44] A. Turhan, K. Keller, J. S. Brenizer, and M. M. Mench. Passive control of liquid water storage and distribution in a PEFC through flow field design. Journal of Power Sources, 180:773-783, 2008.

[45] J. Zhang, D. Kramer, R. Shimoi, Y. Ono, E. Lehmann, A. Wokaun, K. Shinohara, and G. Scherer. In situ diagnostic of two-phase flow phenomena in polymer electrolyte fuel cells by neutron imaging part b - material variations. Electrochimica Acta, 51:2715-2727, 2006.

[46] J. Zhang and R. Datta. Sustained potential oscillations in proton exchange membrane fuel cell with PtRu as anode catalyst. Journal of the Electrochemical Society, 149(11):A1423 - A1431, 2002.

[47] A. Z. Weber and J. Newman. Transport in polymer electrolyte membranes: mathematical model. Journal of Electrochemical Society, 51(2):A311-A325, 2005.

[48] Y. Bultel, K. Wiezell, F. Jaouen, P. Ozil, and G. Lindbergh. Investigation of mass transport in gas diffusion layer at the air cathode of PEMFC. Electrochimica Acta, 51:474 - 488, 2005.

[49] J. Benziger, E. Kimball, R. Mejia-Ariza, and I. Kevekidis. Oxygen mass transport limitations at the cathode of polymer electrolyte membrane fuel cells. AIChe, 57(9):2505-2517, 2011.

[50] M. Lindstrom and B. Wetton. A comparison of Fick and MaxwellStefan diffusion formulations in PEMFC gas diffusion layers. Heat and Mass Transfer, pages 1-8, 2016. 
[51] M. Eikerling, Y. I. Kharkats, A. A. Kornyshev, and Y. M. Volfkovich. Phenomenological theory of electro-osmotic effect and water management in polymer electrolyte proton-conducting membranes. Journal of Electrochemical Society, 145(8):2684-2699, 1998.

[52] T. F. Fuller. PhD Thesis. University of California, Berkeley edition, 1992.

[53] T. A. Zawodzinski, M. Neeman, L. O. Sillerud, and S. Gottesfeld. Determination of water diffusion coefficients in perfluorosulfonate ionomeric membranes. Journal of Physical Chemistry, 95:6040-6044, 1991.

[54] Y. Wang and C. Y. Wang. Simulation of flow and transport phenomena in a polymer electrolyte fuel cell under low-humidity operation. Journal of Power Sources, 147(1-2):148 - 161, 2005.

[55] R. Hanke-Rauschenbach, M. Mangold, and K. Sundmacher. Bistable current-voltage characteristics of PEM fuel cells operated with reduced feed stream humidification. Journal of Electrochemical Society, 155(2):B97 - B107, 2008.

[56] J. P. Corriou. Process control: theory and application. Springer, 2004th edition, 2004.

[57] H. G. Bock, T. Carraro, W. Jäger, S. Körkel, R. Rannacher, and J. P. Sclöder. Model based parameter estimation. Springer, 2013.

[58] B. Sanchez, C. R. Rojas, G. Vandersteen, R. Bragos, and J. Schoukens. 
On the calculation of the d-optimal multisyne excitation power spectrum fot broadband impedance spectroscopy measurements. Measurement Science and Technology, 23:1 - 15, 2012.

[59] K. Vanhoenacker, J. Schoukens, J. Schwevers, and D. Vaes. Summary and comparing overview of techniques for the detections of nonlinear distortion. Proceeding of ISMA 2002, 2:1241 - 1252, 2002.

[60] Q. Mao and U. Krewer. Total harmonic distortion analysis of oxygen reduction reaction in proton exchange membrane fuel cells. Electrochimica Acta, 103:188 - 198, 2013.

[61] J. J. Giner-Sanz, E. M. Ortega, and V. Perez-Herranz. Total harmonic distortion based method for linearity assessment in electrochemical system in the contest of EIS. Electrochimica Acta, 186:598 - 612, 2015.

[62] S. Strahl, A. Husar, and A. A. Franco. Electrode structure effects on the performance of open-cathode proton exchange membrane fuel cells: A multiscale modeling approach. International Journal of $\mathrm{Hy}$ drogen Energy, 39:9752-9767, 2014. 